\title{
Magnetic Resonance Imaging Predictive Model Determines Hamstring Autograft Size for Anterior Cruciate Ligament Reconstruction in Patients Under 18 Years Old
}

\author{
Benjamin Sherman, D.O., Kevin Kwan, B.S., and John Schlechter, D.O.
}

\begin{abstract}
Purpose: To evaluate the predictive value of magnetic resonance imaging in determining hamstring autograft size preoperatively for pediatric anterior cruciate ligament reconstruction. Methods: We conducted an analysis of patients younger than 18 years who underwent quadrupled hamstring autograft anterior cruciate ligament reconstruction. Semitendinosus tendon (ST) and gracilis tendon (GT) cross-sectional areas were measured on preoperative knee magnetic resonance imaging scans. Height, weight, body mass index, and sex were analyzed using ordinal regression analysis. A classification and regression tree (CART) was constructed to identify predictors of the predetermined intraoperative graft size of $8 \mathrm{~mm}$ or greater. Sensitivity and specificity were determined based on specified predictors. Results: This study included 102 patients ( 52 female and 50 male patients). The average age was $15.8 \pm 1.4$ years. At the time of surgery, 38 patients $(37.3 \%)$ had open physes, with an average age of $13.4 \pm 0.96$ years. Of the 102 patients, $55(53.9 \%)$ had 8 -mm or larger grafts, $31(30.4 \%)$ had $7.5-\mathrm{mm}$ grafts, and $16(15.7 \%)$ had $7-\mathrm{mm}$ or smaller grafts. In 12 patients $(31.6 \%)$ with open physes, the graft size was less than $8 \mathrm{~mm}$. Male patients were more likely to have 8 -mm or larger grafts $(P<.001)$, as were patients with a greater height $(170.1 \pm 7.3 \mathrm{~cm}$ vs $161.1 \pm 12.0 \mathrm{~cm}, P=.013)$ and those with a greater weight $(79.7 \pm$ $23.4 \mathrm{~kg}$ vs $57.1 \pm 16.2 \mathrm{~kg}, P=.005)$. For all patients, predictive modeling determined that if the cross-sectional areas of the ST and GT were $31.2 \mathrm{~mm}^{2}$ or greater, then $87.5 \%$ of patients would have a graft size of $8 \mathrm{~mm}$ or greater with an $80 \%$ sensitivity and 74\% specificity. Similarly, if the cross-sectional areas of the ST and GT were $31.4 \mathrm{~mm}^{2}$ or greater in patients with open physes, then $100 \%$ of patients would have a graft size of $8 \mathrm{~mm}$ or greater with a $46 \%$ sensitivity and $99 \%$ specificity. Conclusions: A preoperative summation of the cross-sectional areas of the ST and GT greater than $31 \mathrm{~mm}^{2}$ in patients younger than 18 years with open or closed physes can help the surgeon preoperatively predict which patients will have an intraoperative graft size of $8 \mathrm{~mm}$ or greater. The risk factors for a smaller graft size include female sex, a shorter height, and a lighter weight. Level of Evidence: Level III, retrospective comparative study.
\end{abstract}

$\mathbf{T}$ here is believed to be a rising incidence of anterior cruciate ligament (ACL) injuries in the youth population owing to an increase in athletic participation

From Children's Hospital of Orange County, Orange, California, U.S.A. (B.S., K.K., J.S.); and Riverside University Health System, Moreno Valley, California, U.S.A. (B.S., J.S.).

The authors report the following potential conflicts of interest or sources of funding: J.S. is a paid speaker for Arthrex, outside the submitted work. Full ICMJE author disclosure forms are available for this article online, as supplementary material.

Received August 8, 2020; accepted January 12, 2021.

Address correspondence to Benjamin Sherman, D.O., Riverside University Health System, 26520 Cactus Ave, B2042, Moreno Valley, CA 92555, U.S.A.

E-mail:DrBenjaminSherman@gmail.com

(C) 2021 THE AUTHORS. Published by Elsevier Inc. on behalf of the Arthroscopy Association of North America. This is an open access article under the CC BY-NC-ND license (http://creativecommons.org/licenses/by-nc-nd/4.0/). 2666-061X/201387

https://doi.org/10.1016/j.asmr.2021.01.009 and sports specialization. ${ }^{1,2}$ Early surgical intervention is commonly recommended by experts to prevent secondary chondral and meniscal damage. ${ }^{3}$ Bone-patellar tendon-bone, iliotibial (IT) band, quadriceps, and hamstring autografts are among the common autograft options for pediatric ACL reconstruction (ACLR).

Because of concerns of growth arrest, bone-patellar tendon-bone autograft is less commonly used in skeletally immature patients and the IT band autograft is reserved for only the youngest of pediatric patients requiring femoral and tibial physeal-sparing techniques. Quadriceps tendon reconstruction is a newer technique and has shown good preliminary results; however, there is a paucity of long-term data in the pediatric population. ${ }^{4}$ Hamstring tendon autografts are the most commonly used graft for ACLR in the skeletally immature pediatric population. ${ }^{2,5}$ However, hamstring autografts have come under scrutiny 
recently for being of a smaller size than other grafts, which may lead to higher failure rates. ${ }^{6}$ Recent evidence has suggested that a graft size of less than $8 \mathrm{~mm}$ and age younger than 20 years are the most significant risk factors influencing graft failure. ${ }^{7,8}$

In children or adults with small intraoperative hamstring autografts, the surgeon's options are 4-fold. First, the surgeon can harvest a different autograft, which would increase the morbidity of the operation. Second, the surgeon can accept the small graft size. Third, the surgeon can modify the graft to create a 5- to 8 -strand graft and use an all-inside reconstruction technique. ${ }^{9}$ However, this can be technically challenging and is dependent on the surgeon's abilities, as well as the lengths of the gracilis tendon (GT) and semitendinosus tendon (ST). Finally, the surgeon can augment the autograft with an allograft. Although short-term studies have shown good outcomes with 5and 6-strand graft reconstructions, allograft augmentation results reveal potentially increased failure rates compared with acceptance of the native small graft. ${ }^{10-14}$

However, if the surgeon knows preoperatively that a patient is at risk of having a small hamstring autograft, the surgeon has the additional choice of using an alternative graft. This ongoing problem has led researchers to attempt to predict hamstring autograft size using magnetic resonance imaging (MRI) in the adult population, with promising results; however, there is a paucity of data in patients younger than 18 years. ${ }^{15,16}$

The purpose of this study was to evaluate the predictive value of MRI in determining hamstring autograft size preoperatively for pediatric ACLR. Our hypothesis was that preoperative knee MRI scans could be used to determine an intraoperative hamstring autograft size of $8 \mathrm{~mm}$ or greater for pediatric ACLRs.

\section{Methods}

All patients who had undergone primary ACLR from January 2017 to December 2018 were identified. The inclusion criteria were patients younger than 18 years who underwent surgery using a quadrupled hamstring autograft. Patients were excluded if their preoperative knee MRI scans were not available for viewing on the Synapse picture archiving and communication system (Synapse Workstation, version 4.4.210; FujiFilm Medical Systems USA, Stamford, CT). Additionally, patients were excluded from the study if they underwent a previous ACLR or ipsilateral knee operation or were aged 18 years or older. In all patients, the indication for surgery was symptomatic knee instability with an MRIconfirmed ACL tear.

\section{Surgical Technique for Graft Harvest and Preparation}

The graft harvest was performed using a 2 -cm vertical incision over the pes anserine to harvest both the ST

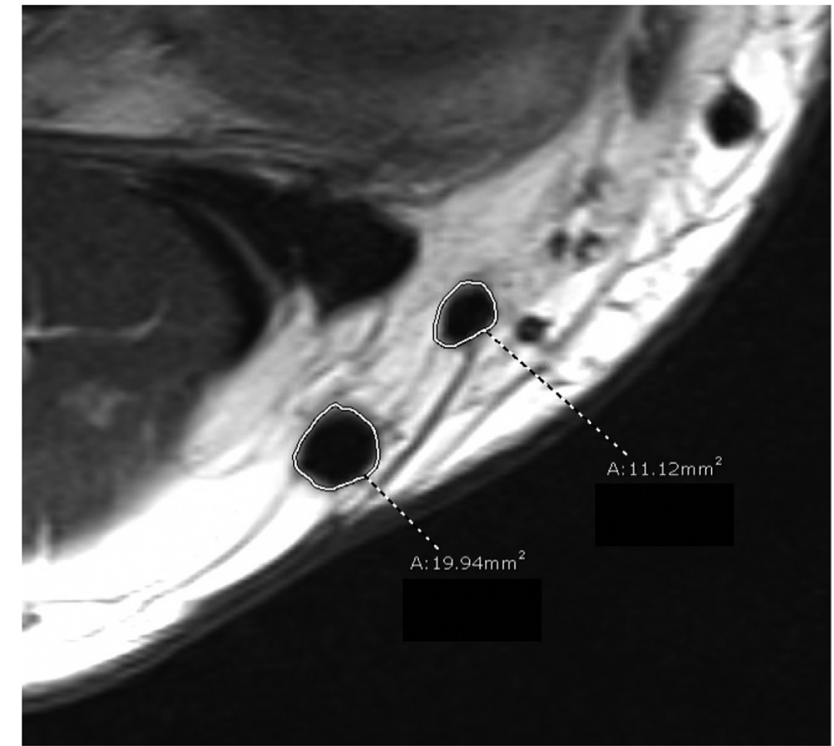

Fig 1. Magnetic resonance imaging of a right knee cross section showing the measurement technique for determining the cross-sectional area (A) of the semitendinosus and gracilis tendons using the field-of-interest tool.

and GT. Each side of the tendons was whipstitched using a FiberLoop suture (Arthrex, Naples, FL). The ST and GT were placed together and folded in half to create a quadrupled hamstring autograft and were then sized using the Arthrex AR-1886 graft-sizing block, which comes in $0.5-\mathrm{mm}$ increments from 4.5 to $12.0 \mathrm{~mm}$. The graft size was determined as the diameter hole that the entire graft could traverse easily. A No. 3-0 absorbable suture was then buried into the graft on each side to prevent sliding, and an Arthrex TightRope device was placed on the femoral side of the graft. The graft was placed onto the tensioning device. All measurements were performed before the graft was tensioned.

\section{Data Collection}

All surgical procedures were performed by a single pediatric sports medicine-trained surgeon (J.S.). Two physicians (a pediatric sports medicine-trained attending orthopaedic surgeon [J.S.] and a postgraduate year 5 orthopaedic resident physician [B.S.]) individually measured the ST-GT cross-sectional areas and diameters using the field of interest and distance measurement tools on axial Tl-weighted MRI cuts (Figs 1 and 2). The slice used for measurement was identified as the largest section by the measuring surgeons. Demographic data (height, weight, body mass index [BMI], and sex) and operative data (graft size) were then collected and recorded.

\section{Statistical Analysis}

Data were analyzed by an independent statistician. Ordinal regression analysis was performed to determine the relation between anthropometric or demographic 


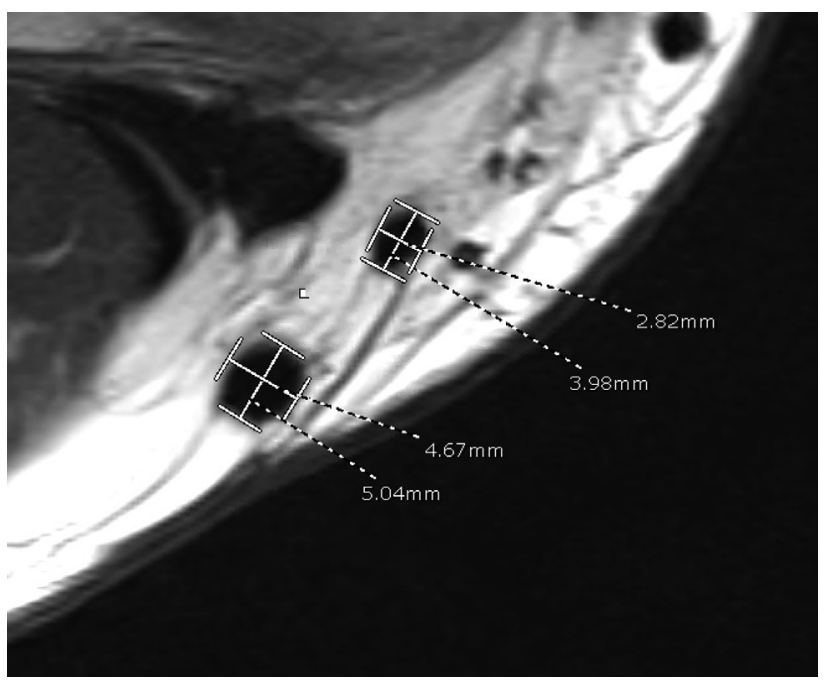

Fig 2. Magnetic resonance imaging of a right knee cross section showing the measurement technique for determining the anteroposterior and mediolateral diameters of the semitendinosus and gracilis tendons.

data and graft size. A classification and regression tree (CART) was then constructed. CART analysis is a technique using computer regression analysis to identify optimal cutoffs and identify complex interactions between predictive variables. The CART algorithm was set to identify predictors of having a graft size of $8 \mathrm{~mm}$ or greater. The data were then separated into those of patients with open physes versus closed physes, and the same statistical tests were run in these subgroups. Receiver operating characteristic (ROC) curve analyses were created to assess the performance of the predictive model and determine sensitivity and specificity. Interrater reliability was determined using the interclass correlation coefficient and classified using the classification of Munro. ${ }^{17}$ All analyses were performed using IBM SPSS Statistics for Windows (version 24.0 [released in 2016]; IBM, Armonk, NY) with $\alpha$ set at $P<.05$ to declare significance.

\section{Results}

During the study period, 146 consecutive patients underwent ACLR. We excluded 30 patients because they underwent ACLR with bone-patellar tendon-bone autograft; 5, with IT band autograft; and 4, with quadriceps tendon autograft. An additional 5 patients were aged 18 years or older and were thus excluded. This study included 102 patients (50 male and 52 female patients) with an average age of $15.7 \pm 1.4$ years (range, 11.8-17.9 years) and average BMI of 26.1 \pm 5.3 (range, 16.0-40.0). Demographic data are summarized in Table 1. The average age of male patients was $15.8 \pm$ 1.2 years, with 28 male patients $(56 \%)$ having open physes. The average age of female patients was $15.7 \pm$ 1.6 years, with 10 female patients $(19.2 \%)$ having open physes. The average height was $165.6 \pm 9.3 \mathrm{~cm}$, and the average graft size was $7.8 \mathrm{~mm}$ (range, 6.0-10.0 mm). Of the patients, $55(53.9 \%)$ had graft sizes of $8 \mathrm{~mm}$ or greater, 31 (30.4\%) had 7.5-mm grafts, and 16 (15.7\%) had graft sizes of $7 \mathrm{~mm}$ or smaller. Open femoral and/or tibial physes were observed in 38 patients $(37.3 \%)$, whereas 64 patients $(62.7 \%)$ had closed physes. Among the patients with open physes, $12(31.6 \%)$ had graft sizes of less than $8 \mathrm{~mm}$ and $26(68.4 \%)$ had graft sizes of $8 \mathrm{~mm}$ or greater. In the combined group (open and closed physes), patients with grafts of $8 \mathrm{~mm}$ or greater had a higher average height $(169.0 \pm 8.6 \mathrm{~cm}$ vs $162.3 \pm$ $9.0 \mathrm{~cm}, P<.001)$ and a greater average weight $(76.9 \pm$ $20.3 \mathrm{~kg}$ vs $67.4 \pm 17.5 \mathrm{~kg}, P=.014)$. Male patients were more likely than female patients to have graft sizes of 8 mm or larger $(P<.001)$.

MRI analysis showed that patients with a graft size of $8 \mathrm{~mm}$ or greater had a greater combined ST-GT crosssectional area $\left(30.5 \pm 5.6 \mathrm{~mm}^{2}\right.$ vs $25.7 \pm 4.8 \mathrm{~mm}^{2}, P<$ $.001)$. Additional MRI analyses are shown in Table 2. Inter-rater reliability was calculated using the interclass correlation coefficient and was found to be 0.68 for determining the cross-sectional areas of the ST and GT. According to the classification of Munro, ${ }^{17}$ this qualifies as moderate reliability. Predictive modeling using the CART analysis was performed and showed that the only variable that met the predictive criteria was the summed cross-sectional area of the ST and GT. Anthropomorphic and demographic data did not have a high enough predictive value to be used in the predictive model. The algorithm determined that if the sum of

Table 1. Demographic Data Comparing Patients by Graft Size $(<8 \mathrm{~mm}$ or $\geq 8 \mathrm{~mm}$ )

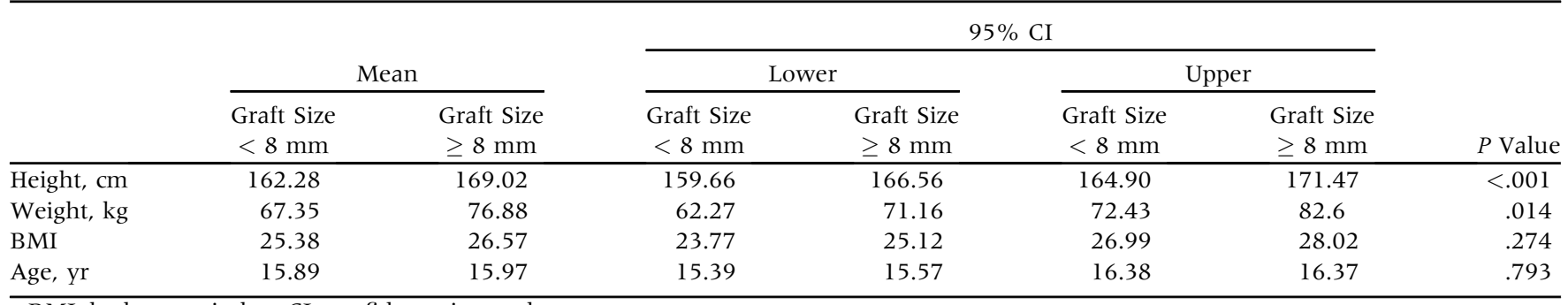

BMI, body mass index; CI, confidence interval. 
Table 2. MRI Measurements Comparing Patients by Graft Size $(<8 \mathrm{~mm}$ or $\geq 8 \mathrm{~mm})$

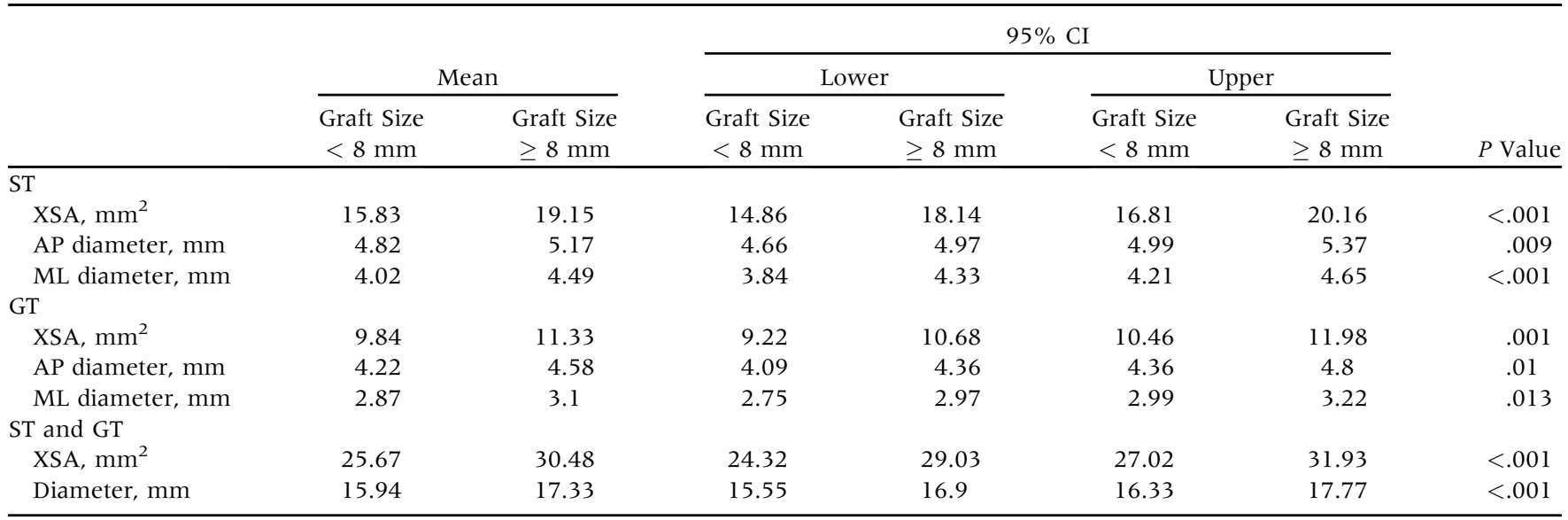

AP, anteroposterior; CI, confidence interval; G, gracilis tendon; ML, mediolateral; MRI, magnetic resonance imaging; ST, semitendinosus tendon; XSA, cross-sectional area.

the cross-sectional areas of the ST and GT was greater than $31.2 \mathrm{~mm}^{2}$, then $87.5 \%$ of patients would have a graft size of $8 \mathrm{~mm}$ or greater. The ROC curve analysis determined the model to have an $80 \%$ sensitivity and $74 \%$ specificity for predicting a graft size of $8 \mathrm{~mm}$ or larger (Fig 3).

\section{Open Physes}

In patients with open physes, the average age was $13.4 \pm 0.96$ years. Patients with a graft size of $8 \mathrm{~mm}$ or greater had a greater average height $(170.1 \pm 7.3 \mathrm{~cm}$ vs $161.1 \pm 12.0 \mathrm{~cm}, P=.013)$, average weight $(79.7 \pm 23.4 \mathrm{~kg}$ vs $57.1 \pm 16.2 \mathrm{~kg}, P=.005)$, and BMI $(26.8 \pm 5.1$ vs $21.8 \pm 5.3, P=.014)$. MRI analysis showed that patients with a graft size of $8 \mathrm{~mm}$ or greater had a greater combined ST-GT cross-sectional area $\left(31.4 \pm 5.0 \mathrm{~mm}^{2}\right.$ vs $\left.27.0 \pm 3.9 \mathrm{~mm}^{2}, P=.013\right)$. Predictive modeling using the CART analysis was performed, and the algorithm determined that if the sum of the cross-sectional areas of the ST and GT was greater than $31.4 \mathrm{~mm}^{2}$, then $100 \%$ of patients would have a graft size of $8 \mathrm{~mm}$ or greater. The ROC curve analysis determined the model to have an $46 \%$ sensitivity and $99 \%$ specificity for predicting a graft size of $8 \mathrm{~mm}$ or larger.

\section{Discussion}

The most important finding of this study is the predictive model that can be easily used by orthopaedic surgeons to help identify which patients are likely to have hamstring autografts of $8 \mathrm{~mm}$ or larger. A preoperative measurement of the cross-sectional area of the ST and GT of $31.2 \mathrm{~mm}^{2}$ predicted a graft size of 8 $\mathrm{mm}$ or greater in $87.5 \%$ of patients younger than 18 years. Similarly, in patients with open physes, a summed measurement of the ST-GT cross-sectional area of $31.4 \mathrm{~mm}^{2}$ predicted a graft size of $8 \mathrm{~mm}$ or greater in
$100 \%$ of patients. In a study of 27 adolescents aged 12 to 18 years, Bickel et al. ${ }^{18}$ measured the axial T1 MRI cross-sectional area of the ST and GT to determine the probability of obtaining an autograft of $7 \mathrm{~mm}$ or greater. They found that if the summation of the cross-sectional areas of both tendons was $18 \mathrm{~mm}^{2}$, there was an $88 \%$ chance of harvesting an autograft of $7 \mathrm{~mm}$ or greater. Our predictive model was unable to predict an autograft

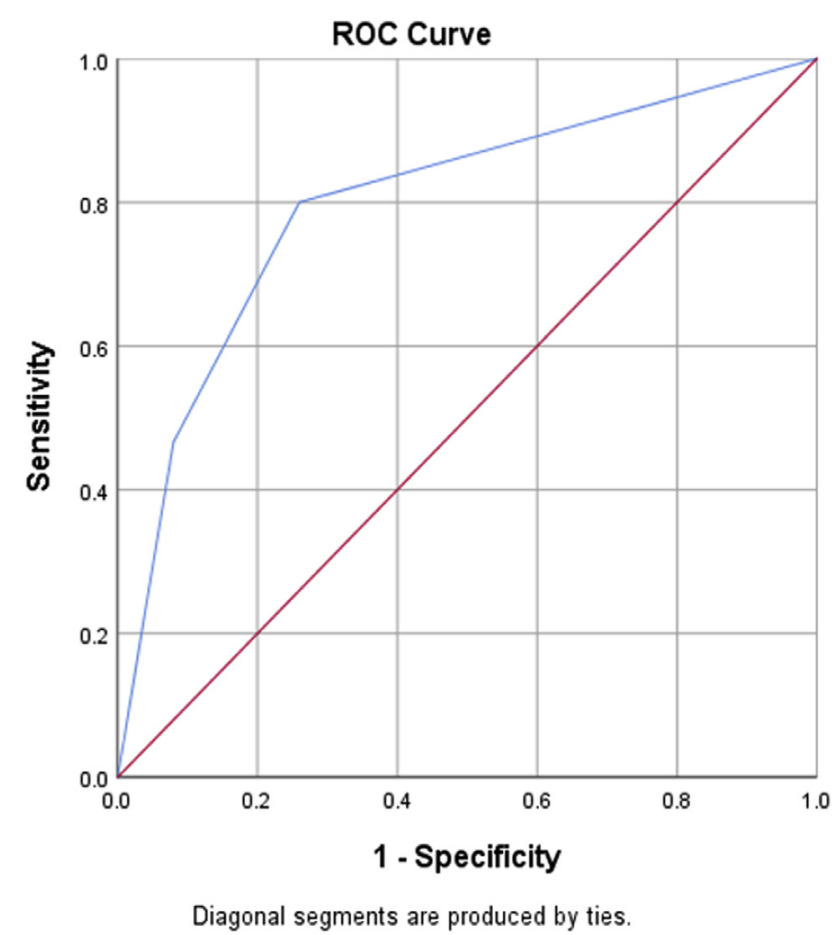

Fig 3. Receiver operator characteristic (ROC) curve of the predictive model for determining a hamstring autograft size of $8 \mathrm{~mm}$ or greater using the sum of the cross-sectional areas of the semitendinosus and gracilis tendons greater than 31.2 $\mathrm{mm}^{2}$. 
size of $7 \mathrm{~mm}$; however, this was because we did not have a large enough sample of patients with grafts under $7 \mathrm{~mm}$. In contrast to the study by Bickel et al., in which $15 \%$ of patients had graft sizes of less than $7 \mathrm{~mm}$, our study included only 5 patients $(5.0 \%)$ who had graft sizes of less than $7 \mathrm{~mm}$.

Additional studies have been performed using MRI to determine autograft size in the adult population. Grawe et al. ${ }^{19}$ measured the MRI cross-sectional area of the ST and GT in 84 skeletally mature patients (average age, 36 years) undergoing ACLR. The study found that a cross-sectional area of $21.64 \mathrm{~mm}^{2}$ was predictive of a graft size of $8 \mathrm{~mm}$. Erquicia et al. ${ }^{20}$ performed a similar study in 33 patients undergoing ACLR: By using $2 \times$ magnification, they found that patients with a measured cross-sectional area greater than $28.1 \mathrm{~mm}^{2}$ had a high likelihood of having a graft of $8 \mathrm{~mm}$ or greater. Similarly to the study by Erquicia et al., we found that a measured cross-sectional area of $31.2 \mathrm{~mm}^{2}$ was needed to predict a graft size of $8 \mathrm{~mm}$ or greater in $87.5 \%$ of patients. This equated to a sensitivity of $80 \%$ and specificity of $74 \%$. The cross-sectional area needed in our study was significantly higher than that in the study by Grawe et al. $\left(31.2 \mathrm{~mm}^{2}\right.$ vs $\left.21.64 \mathrm{~mm}^{2}\right)$. This difference is likely explained by variation in the way different surgeons perform measurements, as well as the locations on axial MRI chosen. Grawe et al. used the largest portion of the femur on axial imaging to determine the location to measure, whereas our study used the location where the tendons were the largest. This difference could explain why the cross-sectional area needed in our study is larger than the cross-sectional area reported by Grawe et al. Additionally, the population in our study differed from that of Grawe et al: Our study comprised patients younger than 18 years (average age, 15.93 years), whereas their study consisted of an adult population with an average age of 36 years. Owing to the younger age in our population and the higher rate of having small grafts inherent in the pediatric population, it is also possible that the crosssectional area needed to predict a graft size of $8 \mathrm{~mm}$ or greater with high certainty is larger in the younger population.

MRI has been studied in the adult population to determine which patient characteristics correlate with increased graft size. Leiter et al. ${ }^{21}$ found a correlation between increased graft size and male sex, as well as increased height, weight, and BMI. Our study, much like several other studies, confirms many of these findings. ${ }^{19,22}$ This study found that increased height, increased weight, and male sex correlated with an increase in graft size. Unlike in previous studies, BMI was not statistically significant in our cohort. However, it is important to note that the study population was an exclusively pediatric population with an average BMI of
25.98 whereas previous studies included predominantly adults.

Pediatric and adolescent patients undergoing ACLR compose the group with the highest risk of graft failure owing to their inherently high activity level and risk of having smaller hamstring autografts compared with the adult population. ${ }^{8}$ This study provides easily understandable and useful data for orthopaedic surgeons treating young patients to improve the surgeons' ability to predict which patients are at high risk of having small hamstring autografts. With this information, surgeons can better plan which ACLR technique to use or can consider selecting an alternative autograft. Although techniques involving multiple strands can be incorporated to mitigate graft failure, a reliable preoperative tool can provide surgeons with information that will aid in preoperative planning and intraoperative decision making.

\section{Limitations}

There are several limitations to this study. In the study population, $37 \%$ of patients had open physes and $63 \%$ had closed physes. This combined population may make the data more difficult to interpret and generalize between the 2 groups. However, the predictive algorithms did find that the combined measurement of the ST and GT needed to be confident in harvesting a graft of $8 \mathrm{~mm}$ or larger was similar between the groups ( 31.2 $\mathrm{mm}^{2}$ vs $31.4 \mathrm{~mm}^{2}$ ). Additionally, the study showed only moderate inter-rater reliability, which may make generalization of the measurements less accurate.

\section{Conclusions}

A preoperative summation of the cross-sectional areas of the ST and GT greater than $31 \mathrm{~mm}^{2}$ in patients younger than 18 years with open or closed physes can help the surgeon preoperatively predict which patients will have an intraoperative graft size of $8 \mathrm{~mm}$ or greater. The risk factors for a smaller graft size include female sex, a shorter height, and a lighter weight.

\section{References}

1. Fabricant PD, Kocher MS. Anterior cruciate ligament injuries in children and adolescents. Orthop Clin North Am 2016;47:777-788.

2. Tepolt FA, Feldman L, Kocher MS. Trends in pediatric ACL reconstruction from the PHIS database. J Pediatr Orthop 2018;38:e490-e494.

3. Smith ID, Irfan A, Huntley JS, Spencer SJ. What is the best treatment for a child with an acute tear of the anterior cruciate ligament? J Paediatr Child Health 2018;54: 1037-1041.

4. Albright J, Lepon AK, Mayer S. Anterior cruciate ligament reconstruction in pediatric and adolescent patients using quadriceps tendon autograft. Sports Med Arthrosc Rev 2016:24:159-169. 
5. Collins MJ, Arns TA, Leroux T, et al. Growth abnormalities following anterior cruciate ligament reconstruction in the skeletally immature patient: A systematic review. Arthroscopy 2016;32:1714-1723.

6. Ho B, Edmonds EW, Chambers HG, Bastrom TP, Pennock AT. Risk factors for early ACL reconstruction failure in pediatric and adolescent patients: A review of 561 cases. J Pediatr Orthop 2018;38:388-392.

7. Conte EJ, Hyatt AE, Gatt CJ Jr, Dhawan A. Hamstring autograft size can be predicted and is a potential risk factor for anterior cruciate ligament reconstruction failure. Arthroscopy 2014;30:882-890.

8. Magnussen RA, Lawrence JT, West RL, Toth AP, Taylor DC, Garrett WE. Graft size and patient age are predictors of early revision after anterior cruciate ligament reconstruction with hamstring autograft. Arthroscopy 2012;28:526-531.

9. Leonard JP. Editorial Commentary: My harvested hamstring autograft is too small. now what? Arthroscopy 2019;35: 1555-1556.

10. Park K, Brusalis CM, Ganley TJ. The 8-strand hamstring autograft in anterior cruciate ligament reconstruction. Arthrosc Tech 2016;5:el105-el109.

11. Tutkus V, Kluonaitis K, Silove S, Tutkuviene J. ACL reconstruction using 5- or 6-strand hamstring autograft provides graft's diameter bigger than $8 \mathrm{~mm}$. Knee Surg Sports Traumatol Arthrosc 2018;26:1349-1356.

12. Richardson MW, Tsouris ND, Hassan CR, et al. A biomechanical comparison of alternative graft preparations for all-inside anterior cruciate ligament reconstruction. Arthroscopy 2019;35:1547-1554.

13. Perkins CA, Busch MT, Christino M, Herzog MM, Willimon SC. Allograft augmentation of hamstring anterior cruciate ligament autografts is associated with increased graft failure in children and adolescents. Am J Sports Med 2019;47:1576-1582.

14. Pennock AT, Ho B, Parvanta K, et al. Does allograft augmentation of small-diameter hamstring autograft ACL grafts reduce the incidence of graft retear? Am J Sports Med 2017;45:334-338.
15. Vardiabasis N, Mosier B, Walters J, Burgess A, Altman G, Akhavan S. Can we accurately predict the quadruple hamstring graft diameter from preoperative magnetic resonance imaging? Orthop J Sports Med 2019;7: 2325967119834504.

16. An VVG, Scholes C, Mhaskar VA, Parker DA, Fritsch BA. Regression modelling combining MRI measurements and patient anthropometry for patient screening and prediction of graft diameter in hamstring autograft arthroscopic ACL reconstruction. Asia Pac J Sports Med Arthrosc Rehabil Technol 2017;8: 24-31.

17. Munro BH. Statistical methods for health care research. Philadelphia: Lippincott Williams \& Wilkins, 2005.

18. Bickel BA, Fowler TT, Mowbray JG, Adler B, Klingele K, Phillips G. Preoperative magnetic resonance imaging cross-sectional area for the measurement of hamstring autograft diameter for reconstruction of the adolescent anterior cruciate ligament. Arthroscopy 2008;24: 1336-1341.

19. Grawe BM, Williams PN, Burge A, et al. Anterior cruciate ligament reconstruction with autologous hamstring: Can preoperative magnetic resonance imaging accurately predict graft diameter? Orthop J Sports Med 2016;4: 2325967116646360.

20. Erquicia JI, Gelber PE, Doreste JL, Pelfort X, Abat F, Monllau JC. How to improve the prediction of quadrupled semitendinosus and gracilis autograft sizes with magnetic resonance imaging and ultrasonography. Am J Sports Med 2013;41:1857-1863.

21. Leiter J, Elkurbo M, McRae S, Chiu J, Froese W, MacDonald P. Using pre-operative MRI to predict intraoperative hamstring graft size for anterior cruciate ligament reconstruction. Knee Surg Sports Traumatol Arthrose 2017;25:229-235.

22. Corey S, Mueller T, Hartness C, Prasad BM. Correlation of intra-operative hamstring autograft size with preoperative anthropometric and MRI measurements. J Orthop 2018;15:988-991. 